\title{
Smartphone-Based Paper Microfluidic Detection of Paraquat Mediated by Citrate-Capped Silver Nanoparticles
}

\section{Navaporn Sritong}

Suranaree University of Technology

\section{Pakawat Kongpreecha}

Suranaree University of Technology

Jeong-Yeol Yoon

The University of Arizona

Sineenat Siri ( $\nabla$ ssinee@sut.ac.th )

Suranaree University of Technology https://orcid.org/0000-0002-2740-5724

\section{Research Article}

Keywords: Colorimetry, Paper microfluidics, Paraquat, Silver nanoparticles, Smartphone detection, $\mu$ PAD

Posted Date: October 19th, 2021

DOl: https://doi.org/10.21203/rs.3.rs-945736/v1

License: (c) This work is licensed under a Creative Commons Attribution 4.0 International License. Read Full License 


\section{Abstract}

Paraquat has been widely used in agriculture due to its efficient herbicidal activity. However, its remaining residues in the environment have caused eco-toxicity to live organisms and humans. Therefore, a portable and sensitive sensing system is required for field-monitoring paraquat towards eco-friendly usage. Toward this goal, citrate-capped silver nanoparticles (AgNPs) on a microfluidic paper analytic device ( $\mu \mathrm{PAD}$ ) were used to detect paraquat, accompanied by smartphone detection. Capillary action through paper fibers enabled the distribution of pre-loaded AgNPs across paper microfluidic channels and allowed rapid citrate-paraquat binding, which led to rapid, reproducible, and sensitive detection of paraquat. Full extents of color changes were recorded within 1 min using a smartphone camera, using only $2 \mu \mathrm{L}$ samples. The assay's linear range was from 2.5 to $20 \mathrm{ppm}$, and the limit of detection was 2.3 ppm $(8.94 \mu \mathrm{M})$ paraquat. Successful selectivity was also demonstrated using several different ions and herbicides, without substantial non-specific aggregation. Moreover, paraquat was further assayed with co-existing ions and field water samples, with satisfactory analytical recoveries of $95-117 \%$, demonstrating its potential application towards on-site and field-ready assay of paraquat.

\section{Introduction}

Paraquat (1,1-dimethyl-4,4-bipyridinium dichloride) is one of the most widely used herbicides because it can control a broad spectrum of broadleaf weeds and grasses in a fast-acting and non-selective manner [1]. Paraquat interferes with an intracellular electron transfer system and leads to a toxic level of reactive oxygen species (ROS) [2], resulting in cell death. Although paraquat can be photochemically decomposed by ultraviolet (UV) radiation and be degraded by microorganisms, such processes are relatively slow thus may not be practical [3]. Paraquat is highly water-soluble but low volatile [4]. Therefore, paraquat can escalate environmental contamination risks, especially in aquatic environments, such as agricultural runoff and industrial wastewater discharges [5]. Accordingly, there are elevated occupational health risks to farmers who have chronically been exposed to paraquat via inhalation or dermal contact [3]. Paraquatcontaminated vegetables and fruits are alternative routes of paraquat exposure to humans. Such residuals cannot be removed from the surfaces of vegetables and fruits by washing with water, therefore accumulated uptake of paraquat can cause long-term health risks in humans [5]. In many countries, paraquat has been banned, especially in the European Union countries, due to its harms to human health [6]. Nevertheless, paraquat has low cost, ease of use, and high herbicidal effect, thus it remains to be used for agricultures in several countries, leading to continuous accumulation in the environments [5]. Therefore, paraquat is still required to be monitored from the environment to not exceed a harmful level to humans.

Many analytical methods have been developed for evaluating paraquat levels in ecosystems. Such analyses include flow injection analysis [4], liquid chromatography-mass spectrometry (LC-MS) [7], highperformance liquid chromatography (HPLC) [8], electrochemical analysis [9], and fluorescence-probe detecting analysis [10]. These methods have generally provided excellent LOD and selectivity for paraquat detection. However, they require expensive laboratory instruments, time-consuming processes, 
and trained technicians, which are inadequate for field and household use. Towards these applications, paraquat detection should be conducted in a user-friendly, on-site, and low-cost manner. Colorimetric detection is one possible solution that can fulfill these needs-specifically, qualitative or semi-qualitative detection by the naked eye can negate the need for laboratory instruments and trained personnel. Several nanoparticles, especially silver nanoparticles (AgNPs), have widely been used for such colorimetric detection, utilizing their unique optical property of surface plasmon resonance (SPR) [11]. The colors of AgNPs change upon their size, shape, and aggregation state, making them suitable optical transducers for colorimetric biosensing [12]. For instant, dispersive AgNPs of approximately 5-70 nm appear in yellow color but their color changed to brown under aggregation $[13,14]$. AgNPs can be conjugated with specific functional groups for detecting various chemicals and microbes, such as metal ions [15, 16], bacteria [17], pesticides [18, 19], and biomedical markers [20-22]. Previously, AgNP-based paraquat detection was demonstrated through the cyclen dithiocarbamate (DTC) conjugated AgNPs, with a limit of detection (LOD) of $7.21 \mu \mathrm{M}(1.85 \mathrm{ppm})$ paraquat. However, this method still required expensive DTC chemical and a laboratory spectrophotometer [23]. Alternatively, citrate-capped nanoparticles were utilized to detect paraquat, i.e., without using any additional functionalization [5]. In that work, silica gel was added as an adsorbent for purification and pre-concentration purposes. While lower LOD was demonstrated with this method $-0.015 \mathrm{ppm}$ paraquat-it required time-consuming steps of sample purification and preconcentration, had difficulty in differentiating color, and needed a laboratory spectrophotometer. Later, the imidacloprid-capped AgNPs (Imida-AgNPs) was developed for paraquat detection, with the LOD of 6.27 $\mu \mathrm{M}$ (1.61 ppm). However, this method still needed a UV-Vis spectrophotometer [24]. Therefore, we can identify a gap in paraquat detection research, which can demonstrate portable and easy-to-use system as well as satisfactory LOD.

A paper-based microfluidic analytical device $(\mu P A D)$ has gained significant research interest for a portable platform due to its low cost, small sample volume, ease of use, and paper's innate filtration capability [23]. In a $\mu$ PAD system, samples and reagents flow through paper fibers via capillary action and allow the colorimetric dyes to spread out through the hydrophilic channel, leading to high optical contrast. This benefit makes it possible to evaluate the color changes of nanoparticle-based assays on $\mu$ PADs rather than using a cuvette and a UV-Vis spectrophotometer. In addition, such color changes can be quantified by digital imaging devices, including a smartphone [25].

Herein, the smartphone detection of paraquat on a $\mu$ PAD using citrate-capped Ag NPs is proposed as an alternative paraquat assay towards a cost-effective, portable, and easy-to-use sensor. This work is the first report on paraquat detection using AgNPs on $\mu$ PADs with smartphone imaging to the best of our knowledge. The interaction between paraquat and citrate-capped AgNPs could induce aggregation of AgNPs, resulting in color changes that could be correlated to the paraquat concentration [5]. In this work, a smartphone was used to capture the color changes, in which the images were split into red, green, and blue (RGB) channels, and the red absorbance values were evaluated using green and blue as references. LOD and selectivity were evaluated for assaying paraquat from various water samples (with co-existing ions and field water samples). 


\section{Materials And Methods \\ Chemicals}

Autoclaved deionized (DI) water was used throughout the whole experiment. Silver nitrate, sodium borohydride, paraquat dichloride, difenzoquat methyl sulfate, and chlorpyrifos-methyl were purchased from ChemCruz (Dallas, TX, USA). Trisodium citrate dihydrate, sodium sulfate, sodium phosphate dibasic, sodium chloride, and magnesium chloride were obtained from Sigma-Aldrich (St. Louis, MO, USA). Sodium nitrate and calcium chloride dihydrate were purchased from Alfa Aesar (Ward Hill, MA, USA) and Calbiochem (Darmstadt, Germany), respectively.

\section{Synthesis and Characterization of Citrate-capped AgNPs}

To obtained citrate-capped AgNPs, the classical citrate reduction method was used with some modifications [26]. Briefly, $1 \mathrm{mM}$ silver nitrate $(20 \mathrm{~mL})$ was mixed with $1 \mathrm{mM}$ trisodium citrate $(5 \mathrm{~mL})$, followed by adding $25 \mathrm{~mL}$ DI water. Then, $2.7 \mathrm{mM}$ freshly prepared sodium borohydride $(10 \mathrm{~mL})$ was added dropwise under vigorous stirring for $30 \mathrm{~min}$. We adjusted the $\mathrm{pH}$ of the AgNP solution to 10.5 following the previous study [5]. The UV-Vis spectrophotometer (Ocean Optics, Dunedin, FL, USA) was used to monitor the formation of citrate-capped AgNPs by measuring the absorbance at $300-900 \mathrm{~nm}$. The synthesized AgNPs were stored at $4^{\circ} \mathrm{C}$ until used.

The morphology and size of the synthesized AgNPs were analyzed by transmission electron microscope (TEM; Tecnai G2 20 S-Twin, FEl, Hillsboro, OR, USA) with the operating at accelerating voltage of $200 \mathrm{kV}$. The samples were prepared by dropping the colloidal AgNPs on a carbon-coated copper holder. After airdrying at room temperature, the samples were observed under TEM operating at $200 \mathrm{kV}$. The TEM images were analyzed by ImageJ software (National Institutes of Health, Bethesda, MD, USA) and OriginPro 2015 software (OriginLab Corp., Northampton, MA, USA) to determine the particle size and its distribution, respectively. Furthermore, the crystalline nature of AgNPs was analyzed by selected area electron diffraction (SAED) using a Tecnai G2 S-Twin TEM operating at $200 \mathrm{kV}$ using $\mathrm{LaB}_{6}$ filament. All TEM images were taken by a Gatan Orius 200 CCD Camera (Gatan, Pleasanton, CA, USA), equipped with the TEM.

\section{Fabrication of $\mu P A D$}

The $\mu$ PAD fabrication process is illustrated in Fig. 1a. The wax printing method was used to fabricate the paper chips, and their pattern was designed using SolidWorks (Dassault Systemes, SolidWorks Corporation, Waltham, MA, USA). The 2-mm wide and 30-mm long channel designs were printed on the nitrocellulose substrate (GE Healthcare, UK) using a wax printer (ColorQube 8580, Xerox, Wilsonville, OR, USA) to create the hydrophobic barrier. Each chip consisted of three channels to perform three replicates of the assay. The printed chips were cut and heated at $130^{\circ} \mathrm{C}$ on a hot plate until the wax was completely melted and filled the paper pores through the depth of paper substrate within the wax pattern $(<120 \mathrm{~s})$. The chips were then kept in a sealed container under dry and dark conditions until used. 


\section{Assay Procedure and Image Processing}

Paper-based paraquat assays were performed by pre-loading $2 \mu \mathrm{L}$ of $1 \mathrm{mM}$ AgNPs on one end of the $\mu P A D$ channel, which was diffused through the channel via capillary action. The resulting $\mu$ PADs were airdried for $50 \mathrm{~s}$ to get a damp-dried state. For quantitative analysis, $2 \mu \mathrm{L}$ of various concentrations $(0-40$ $\mathrm{ppm}$ ) of paraquat dichloride was subsequently added to the channel at the same position, where the AgNPs were loaded. Within $1 \mathrm{~min}$, paraquat diffused through the channel via capillary action, and the images of the channels were taken using Samsung Galaxy S8 (Samsung Electronics, Suwon, South Korea). The smartphone was placed perpendicular to a paper chip at a $15.5 \mathrm{~cm}$ distance, as depicted in Fig. 1b. The images were taken every $10 \mathrm{~s}$ for 1 min under ambient lighting conditions, using Lapse It application (Interactive Universe, Sao Paulo, Brazil) to lock the focus, white balance, and shutter speed at constant values. All images were transferred to a computer and split into their red, green, and blue channel components by using ImageJ software. At each paraquat concentration, the experiments were performed in three replicates to obtain the mean RGB intensities. Colorations were quantified using red-toblue $(R / B)$ intensity ratios and red-to-green $(R / G)$ intensity ratios. As previously reported, red intensity changes the most upon AgNP aggregation [9], and blue and green intensities serve as references to address the biases in paper color and ambient lighting. These data (normalized red intensities, $I$ ) were then converted to absorbance $(A)$ values using $A=\log \left(I_{0} / I\right)$ [27], where $I_{0}$ is the intensity of blank (AgNPs in DI water). Both the normalized intensities (R/B and R/G) and their absorbance values were plotted against the paraquat concentration to construct suitable calibration curves. The linear fit of these curves provided $R^{2}$ values and slopes. The limit of detection (LOD) was defined as $3 \times \sigma_{\text {blank }} /$ slope, where $\sigma_{\text {blank }}$ is the standard deviation of the blank signals $(n=10)$ and the slope is that of the calibration curve [28]. Selectivity was determined by testing with cations $\left(\mathrm{Ca}^{2+}\right.$ and $\left.\mathrm{Mg}^{2+}\right)$, anions $\left(\mathrm{Cl}^{-}, \mathrm{NO}_{3}{ }^{-}, \mathrm{SO}_{4}{ }^{2-}\right.$, and $\mathrm{PO}_{4}{ }^{3-}$ ), and the positively charged pesticides (difenzoquat and paraquat).

\section{Detection of Paraquat-Spiked Water Samples}

As a potential application, paraquat was spiked into the reclaimed wastewater and river water samples. Reclaimed wastewater was collected from the reclaimed wastewater supply at the University of Arizona campus (Tucson, Arizona, USA) and river water from the Sabino Creek in the Coronado National Forest (Pima, Arizona, USA). The final concentrations of spiked paraquat were 5 and $10 \mathrm{ppm}$ in these field water samples. The assay procedure was identical to the one described in section 2.4. $I_{0}$ was collected using the reclaimed wastewater or the river water without paraquat spiked.

\section{Statistical Analysis}

All data were displayed as means \pm standard deviations. Three replicates were used for each experimental condition, each time on a different microfluidic channel. One-way ANOVA and Tukey's Honestly Significant Differences were used to compare means among groups using SPSS software (SPSS, Chicago, IL, USA). The $p$-value of less than 0.05 represented the statistically significant difference. 


\section{Results And Discussion}

\section{Characterization of AgNPs}

AgNPs were synthesized by the citrate reduction method. The formation of AgNPs could be observed from the reaction-color changes from colorless to brown and finally deep yellow. Also, the formation of citrate-capped AgNPs was confirmed by the presence of a surface plasmon resonance (SPR) peak at 418 $\mathrm{nm}$ (Fig. 2a). The TEM image indicated the uniform dispersion and spherical shape of the synthesized AgNPs (Fig. 2b). Their size distribution was in the range of 6.4-13.6 nm (Fig. 2C), and an average diameter was $9.8 \pm 1.9 \mathrm{~nm}$ as measured from 500 random-picked nanoparticles, indicating uniform and narrow-sized distribution [27]. Additionally, the identity of the synthesized AgNPs was further analyzed by TEM-SAED. The appearance of four diffraction rings confirmed the crystalline nature of the synthesized AgNPs indexed as [111], [200], [220], and [311] planes (Fig. 2d), which were in good agreement with the characteristic of face-centered cubic (fcc) structure of silver [29].

\section{Spectral Analysis of Paraquat-induced AgNP Aggregation}

The interaction between citrate-capped AgNPs and paraquat is based on Coulombic attraction [5]. Paraquat possesses quarternary amines at both ends of the molecule. It can bridge two or more AgNPs together (or decrease the interparticle distance) through binding with citrate groups, as shown in Fig. 3a. Also, with its planar geometry and less steric hindrance structure, paraquat can easily access the surface of AgNPs and induce their aggregation [2,5]. Thus, the colors of AgNP-suspension were changed from yellow to brown colors, when paraquat was added. The dispersed and aggregated forms of AgNPs were monitored using UV-Vis spectra (Fig. 3b). The spectrum of the dispersed AgNPs showed the characteristic SPR peak of silver at $418 \mathrm{~nm}$ [30]. With the presence of paraquat, the SPR peak at $418 \mathrm{~nm}$ was decreased in a dose-dependent response and the induction of absorbance intensity in the wavelength region of 550$800 \mathrm{~nm}$. The observed colors were changed from yellow to yellow-grey. The extent of AgNP-aggregation was dependent upon the paraquat concentration, which spectral responses can quantify, i.e., increase in the redshift range intensity and decrease in the SPR band [31].

\section{Smartphone-based Paraquat Assay on $\mu$ PAD}

The fabricated $\mu \mathrm{PAD}$ was tested for paraquat detection. Various concentrations of paraquat solutions were dropped into the paper chips pre-loaded with citrate-capped AgNPs. The initial color of the AgNPsloaded paper channels was yellow. After adding paraquat, the color of AgNPs was changed from yellow to light greenish-grey, finally grey, with increasing the paraquat concentration from 0 to $40 \mathrm{ppm}$ (Fig. 4a). Sample solutions filled the entire channel after $30 \mathrm{~s}$, and the color was entirely changed within $1 \mathrm{~min}$. Color photographs of $\mu$ PAD were captured using a smartphone and split into three different color channels (RGB) in greyscale using ImageJ software. Three different color intensities were evaluated from each channel. As increasing paraquat concentration, the red and green intensities decreased, and the blue intensity increased. These changes were well correlated with the changes in UV-Vis spectra described in the previous section, i.e., decrease in blue (400-500 nm) absorbance [32], and increases in green (500-580 
$\mathrm{nm})$ [33] and red (580-700 nm) absorbance [28, 34], as absorbance is inversely related to light intensity. Prior to calculating absorbance, the ratios of color intensities were evaluated to address sample-tosample and chip-to-chip variations. The ratios of red over blue (R/B) or red over green (R/G) intensities were plotted against the paraquat concentrations, as shown in Fig. 4b. A stronger correlation was found with $\mathrm{R} / \mathrm{B}$ rather than $\mathrm{R} / \mathrm{G}$, which corresponded to the spectral analysis. Therefore, smartphone-based detection was suitable for determining the color intensity changes of AgNPs, and the R/B intensity ratio was used to calculate the absorbance values.

\section{Assay Characterization: LOD and Selectivity}

Various concentrations of paraquat were added in the pre-loaded AgNPs- $\mu$ PAD, and the color changes were quantified by measuring R/B intensities acquired by a smartphone. These R/B intensities were converted to R/B absorbances using the blank R/B intensities. The R/B absorbances were plotted against the paraquat concentration (Fig. 5), showing the linear response from 2.5 to $20.0 \mathrm{ppm}$ with excellent linearity $\left(R^{2}=0.9980\right)$. We can widen the linear range by increasing the amount of pre-loaded AgNPs, at the cost of sacrificing LOD. The calculated LOD $\left(3 \times \sigma_{\text {blank }} /\right.$ slope) was $2.3 \mathrm{ppm}(8.94 \mu \mathrm{M}$; approximately $\left.10^{-7} \mathrm{M}\right)$. Distinct color changes could be observed with the naked eye at $10 \mathrm{ppm}(3.89 \mu \mathrm{M})$. While Siangproh and colleagues [5] demonstrated a lower LOD of $10^{-8} \mathrm{M}$ than our work for paraquat detection, it had several limitations. These include high time-consuming processes, complex sample preparations, complicated data analyses, and laboratory instrument requirements; all impede its use in fields and households. Our smartphone- and paper microfluidic method could circumvent these limitations and be used for field and household applications. Besides, our developed system covers the risk levels of paraquat to farmers and consumers, $20-40$ ppm, recommended by the United States Environmental Protection Agency (EPA) [35].

To evaluate the selectivity of this assay, we tested the solutions of other foreign ions and other positively charged pesticides. According to Fig. 6 , the R/B intensities of 10 and $20 \mathrm{ppm}$ paraquat were significantly different from control and difenzoquat, which showed the analogous structure with paraquat. As compared with paraquat, difenzoquat is less water-soluble and contains a single positively-charged site, thus making a weaker interaction with the citrate-capped AgNPs [5]. The developed $\mu$ PAD detection assay for paraquat at the concentration of $20 \mathrm{ppm}$ showed a significantly different compared to the other tested chemicals. However, the R/B intensity of $10 \mathrm{ppm}$ paraquat exhibited a non-significant signal from those of $\mathrm{Ca}^{2+}$ and $\mathrm{Cl}^{-}$, potentially indicating the non-specific aggregation of AgNPs. Nevertheless, the concentration of foreign species used in this work $(100 \mathrm{ppm})$ is significantly higher than the typical amounts found in water samples. Thus, their effects on paraquat assay could be minimum. AgNPs have been reported to aggregate in the presence of $\mathrm{Ca}^{2+}$ and $\mathrm{Cl}^{-}$, and these two ions are commonly used in nanoparticle-based detection to improve the LOD [36, 37]. Assays were repeated for detecting $10 \mathrm{ppm}$ paraquat with coexisting ions, and the results are summarized in Table 1. The concentrations of foreign ions were determined from the upper limits of each ion typically found in tap water in the United States [38]. The results show that the coexisting foreign ions did not significantly change the R/B intensities (less than $\pm 5 \%$ ) compared to the assays without coexisting ions. 
Table 1

Effect of coexisting ions on the R/B intensity for the detection of $10 \mathrm{ppm}$ paraquat.

\begin{tabular}{|lll|}
\hline $\begin{array}{l}\text { Coexisting } \\
\text { ions }\end{array}$ & $\begin{array}{l}\text { Concentration } \\
(\mathrm{ppm})\end{array}$ & $\begin{array}{l}\text { \% difference in R/B intensities from those without coexisting } \\
\text { ions }\end{array}$ \\
\hline $\mathrm{Ca}^{2+}$ & 50 & $-2.26 \%$ \\
\hline $\mathrm{Mg}^{2+}$ & 20 & $-2.86 \%$ \\
\hline $\mathrm{Cl}^{-}$ & 25 & $-3.98 \%$ \\
\hline $\mathrm{NO}_{3}{ }^{-}$ & 0.5 & $+3.19 \%$ \\
$\mathrm{SO}_{4}{ }^{2-}$ & 70 & $-2.71 \%$ \\
$\mathrm{PO}_{4}{ }^{3-}$ & 0.1 & $-0.34 \%$ \\
\hline
\end{tabular}

\section{Assay for Field Water Samples}

The developed assay was further tested with paraquat-spiked field water samples. We spiked paraquat into the wastewater at the University of Arizona campus and the river water from the Coronado National Forest at the concentrations of 5 and $10 \mathrm{ppm}$. The results in Table 2 demonstrated that the analytical recovery rates of 5 and $10 \mathrm{ppm}$ paraquat were $105.4 \%$ and $94.61 \%$, respectively, in the reclaimed wastewater. In river water samples, the recovery percentages were $113.9 \%$ and $117.2 \%$ for assaying 5 and $10 \mathrm{ppm}$ paraquat, respectively. These recovery values were within the acceptable range for the spiked recovery experiment, from $70-120 \%$ [39], indicating successful paraquat detection from field water samples.

Table 2

Paraquat assay for field water samples.

\begin{tabular}{|llll|}
\hline Water sample & \multicolumn{2}{c}{ Paraquat (ppm) } & \multirow{2}{*}{ \% Recovery } \\
\cline { 2 - 3 } & Added & Found & \\
\hline Reclaimed wastewater & 5.0 & $5.3 \pm 0.6$ & 105.4 \\
\hline River water & 10.0 & $9.5 \pm 0.4$ & 94.6 \\
& 5.0 & $5.7 \pm 0.4$ & 113.9 \\
\hline & 10.0 & $11.3 \pm 1.1$ & 117.2 \\
\hline
\end{tabular}

\section{Conclusion}

The smartphone-based detection of paraquat using citrate-capped AgNPs on $\mu$ PAD was reported for the first time. The pre-loaded AgNPs are distributed throughout the channel via capillary action, contributing to the reproducibility of the assay, as evidenced by the small standard deviations throughout the assays. 
The citrate-capped AgNPs are aggregated with paraquat via Coulombic attraction, resulting in the color shift from yellow to grey. The capillary flow enhanced the citrate-paraquat binding through paper fibers, generating the assay results in less than $1 \mathrm{~min}$. A small volume of sample $(2 \mu \mathrm{L})$ was necessary to conduct the developed assay. A smartphone camera captured these color changes on $\mu$ PAD under ambient lighting conditions. The normalized light intensities and absorbance values were evaluated. The calibration curve of R/B absorbances against paraquat concentrations was constructed, with the working linear range of 2.5-20 ppm with the LOD of $2.3 \mathrm{ppm}(8.94 \mu \mathrm{M})$. Selectivity experiments with coexisting ions and the assays of paraquat-spiked field water samples corroborated this method's field applicability. Additionally, using a paper platform and a smartphone allows cost-effective, portable, rapid, and reliable paraquat detection assay in various environmental water samples.

\section{Declarations}

Authors Contributions We declare that we contributed significantly towards the research study.Navaporn Sritong: experiment, data analysis, and article-draft. Pakawat Kongpreecha: article-draft. Jeong-Yeol Yoon: experimental design, discussion, editing, and corresponding author. Sineenat Siri: experimental design, discussion, editing, funding acquisition, and corresponding author. All the authors read and approved the manuscript.

Funding This work was supported by Suranaree University of Technology (SUT) and by Thailand Science Research and Innovation (TSRI).

Data Availability The data that support the findings of this study are available from the corresponding author upon reasonable request.

EthicalApproval Not applicable.

Consent to Participate Not applicable.

Consent to Publish Not applicable.

Competing Interests The authors declare no competing interests.

\section{References}

1. Huang, Y., Zhan, H., Bhatt, P.,\& Chen, S. (2019). Paraquat degradation from contaminated environments: Current achievements and perspectives. Frontiers in Microbiology, 10, 1754. https://doi.org/10.3389/fmicb.2019.01754.

2. Kongpreecha, P.,\& Siri, S. (2021). Simple colorimetric screening of paraquat residue in vegetables evaluated by localized surface plasmon resonance of gold nanoparticles. Biotechnology and Applied Biochemistry, 1-11. https://doi.org/10.1002/bab.2191. 
3. Tsai, W.-T. (2013). A review on environmental exposure and health risks of herbicide paraquat. Toxicological \& Environmental Chemistry, 95(2), 197-206.

4. Chuntib, P.,\& Jakmunee, J. (2015). Simple flow injection colorimetric system for determination of paraquat in natural water. Talanta, 144, 432-438.

5. Siangproh, W., Somboonsuk, T., Chailapakul, O.,\& Songsrirote, K. (2017). Novel colorimetric assay for paraquat detection on-silica bead using negatively charged silver nanoparticles. Talanta, 174, 448453.

6. Walsh, A.,\& Kingwell, R. (2021). Economic implications of the loss of glyphosate and paraquat on Australian mixed enterprise farms. Agricultural Systems, 193, 103207. https://doi.org/10.1016/j.agsy.2021.103207.

7. Pizzutti, I. R., Vela, G. M., de Kok, A., Scholten, J. M., Dias, J. V., Cardoso, C. D., Concenço, G.,\& Vivian, R. (2016). Determination of paraquat and diquat: LC-MS method optimization and validation. Food Chemistry, 209, 248-255.

8. Sha, O., Wang, Y., Chen, X.-B., Chen, J.,\& Chen, L. (2018). Determination of paraquat in environmental water by ionic liquid-based liquid phase extraction with direct injection for HPLC. Journal of Analytical Chemistry, 73(9), 862-868.

9. Tagne, R. F. T., Ndifor-Angwagor, N. G., Temgoua, R. C. T., Tchuifon, D. R. T., Vintila, T., Ngueabouo, A. S.,\& Anagho, S. G. (2021). Development of an electroanalytical method using activated rice huskderived carbon for the detection of a paraquat herbicide. Carbon Trends, 4, 100060. https://doi.org/10.1016/j.cartre.2021.100060.

10. Pourghobadi, Z., Makanali, H.\& Zare, H. (2021). Highly sensitive fluorescent probe for detection of paraquat based on nanocrystals. Journal of Fluorescence, 31(2), 559-567.

11. Mohite, P., Apte, M., Kumar, A. R.,\& Zinjarde, S. (2016). Biogenic nanoparticles from Schwanniomyces occidentalis NCIM 3459: Mechanistic aspects and catalytic applications. Applied Biochemistry and Biotechnology, 179(4), 583-596.

12. Yakoh, A., Rattanarat, P., Siangproh, W.,\& Chailapakul, O. (2018). Simple and selective paper-based colorimetric sensor for determination of chloride ion in environmental samples using label-free silver nanoprisms. Talanta, 178, 134-140.

13. Che Sulaiman, I. S., Chieng, B. W., Osman, M. J., Ong, K. K., Rashid, J. I. A., Wan Yunus, W. M. Z., Noor, S. A. M., Kasim, N. A. M., Halim, N. A.,\& Mohamad, A. (2020). A review on colorimetric methods for determination of organophosphate pesticides using gold and silver nanoparticles. Microchimica Acta, 187(2), 131. https://doi.org/10.1007/s00604-019-3893-8.

14. Raza, M. A., Kanwal, Z., Rauf, A., Sabri, A. N., Riaz, S.,\& Naseem, S. (2016). Size-and shape-dependent antibacterial studies of silver nanoparticles synthesized by wet chemical routes. Nanomaterials, 6(4), 74.https://doi.org/10.3390/nano6040074.

15. Divsar, F., Habibzadeh, K., Shariati, S.,\& Shahriarinour, M. (2015). Aptamer conjugated silver nanoparticles for the colorimetric detection of arsenic ions using response surface methodology. Analytical Methods, 7(11), 4568-4576. 
16. Sung, H. K., Oh, S. Y., Park, C.,\& Kim, Y. (2013). Colorimetric detection of $\mathrm{Co}^{2+}$ ion using silver nanoparticles with spherical, plate, and rod shapes. Langmuir, 29(28), 8978-8982.

17. Mocan, T., Matea, C. T., Pop, T., Mosteanu, O., Buzoianu, A. D., Puia, C., Iancu, C.,\& Mocan, L. (2017). Development of nanoparticle-based optical sensors for pathogenic bacterial detection. Journal of Nanobiotechnology, 15(1), 25-38.

18. Ivrigh, Z. J.-N., Fahimi-Kashani, N.,\& Hormozi-Nezhad, M. R. (2017). Aggregation-based colorimetric sensor for determination of prothioconazole fungicide using colloidal silver nanoparticles (AgNPs). Spectrochimica Acta Part A: Molecular and Biomolecular Spectroscopy, 187, 143-148.

19. Shrivas, K., Nirmalkar, N., Ghosale, A.,\& Thakur, S. S. (2016). Application of silver nanoparticles for a highly selective colorimetric assay of endrin in water and food samples based on stereoselective endo-recognition. RSC Advances, 6(35), 29855-29862.

20. Gao, Y., Wu, Y.,\& Di, J. (2017). Colorimetric detection of glucose based on gold nanoparticles coupled with silver nanoparticles. Spectrochimica Acta Part A: Molecular and Biomolecular Spectroscopy, 173, 207-212.

21. Detsri, E.,\& Seeharaj, P. (2017). Colorimetric detection of glutathione based on phthalic acid assisted synthesis of silver nanoparticles. Colloids and Surfaces A: Physicochemical and Engineering Aspects, 533, 125-132.

22. Rostami, S., Mehdinia, A., Jabbari, A., Kowsari, E., Niroumand, R.,\& Booth, T. J. (2018). Colorimetric sensing of dopamine using hexagonal silver nanoparticles decorated by task-specific pyridinum based ionic liquid. Sensors and Actuators B: Chemical, 271, 64-72.

23. Rohit, J. V.,\& Kailasa, S. K. (2014). Cyclen dithiocarbamate-functionalized silver nanoparticles as a probe for colorimetric sensing of thiram and paraquat pesticides via host-guest chemistry. Journal of Nanoparticle Research, 16(11), 2585. https://doi.org/10.1007/s11051-014-2585-x.

24. Ali, S., Shah, M. R., Hussain, S., Khan, S., Latif, A., Ahmad, M.,\& Ali, M. (2021). A facile approach based on functionalized silver nanoparticles as a chemosensor for the detection of paraquat. Journal of Cluster Science. https://doi.org/10.1007/s10876-021-01978-w.

25. Sriram, G., Bhat, M. P., Patil, P., Uthappa, U. T., Jung, H.-Y., Altalhi, T., Kumeria, T., Aminabhavi, T. M., Pai, R. K.,\& Kurkuri, M. D. (2017). Paper-based microfluidic analytical devices for colorimetric detection of toxic ions: A review. TrAC Trends in Analytical Chemistry, 93, 212-227.

26. Ma, S., He, J., Guo, M., Sun, X.,\& Zheng, M. (2016). Facile colorimetric detection of 6benzylaminopurine based on $\mathrm{p}$-aminobenzenethiol functionalized silver nanoparticles. RSC Advances, 6(108), 106608-106614.

27. Amirjani, A.,\& Fatmehsari, D. H. (2018). Colorimetric detection of ammonia using smartphones based on localized surface plasmon resonance of silver nanoparticles. Talanta, 176, 242-246.

28. Meelapsom, R., Jarujamrus, P., Amatatongchai, M., Chairam, S., Kulsing, C.,\& Shen, W. (2016). Chromatic analysis by monitoring unmodified silver nanoparticles reduction on double layer microfluidic paper-based analytical devices for selective and sensitive determination of mercury (II). Talanta, 155, 193-201. 
29. Agnihotri, S., Mukherji, S.,\& Mukherji, S. (2014). Size-controlled silver nanoparticles synthesized over the range 5-100 nm using the same protocol and their antibacterial efficacy. RSC Advances, 4(8), 3974-3983.

30. Chumpol, J.,\& Siri, S. (2018). Simple green production of silver nanoparticles facilitated by bacterial genomic DNA and their antibacterial activity. Artificial Cells, Nanomedicine, and Biotechnology, 46(3), 619-625.

31. Vilela, D., González, M. C.,\& Escarpa, A. (2012). Sensing colorimetric approaches based on gold and silver nanoparticles aggregation: chemical creativity behind the assay. A review. Analytica Chimica Acta, 751, 24-43.

32. Holzman, D. C. (2010). What's in a color? The unique human health effects of blue light. Environmental Health Perspectives, 118(1), A22-A27.

33. Johkan, M., Shoji, K., Goto, F., Hahida, S.,\& Yoshihara, T. (2012). Effect of green light wavelength and intensity on photomorphogenesis and photosynthesis in Lactuca sativa. Environmental and Experimental Botany, 75, 128-133.

34. Gawne, T. J., Siegwart Jr, J. T., Ward, A. H.,\& Norton, T. T. (2017). The wavelength composition and temporal modulation of ambient lighting strongly affect refractive development in young tree shrews. Experimental Eye Research, 155, 75-84.

35. Roberts, J. R.,\& Reigart, J. R. (2013). Recognition and management of pesticide poisonings. US Environmental Protection Agency, Washington, USA.

36. Busayapongchai, P.,\& Siri, S. (2017). Sensitive detection of estradiol based on ligand binding domain of estrogen receptor and gold nanoparticles. Analytical Biochemistry, 518, 60-68.

37. Huynh, K. A.,\& Chen, K. L. (2011). Aggregation kinetics of citrate and polyvinylpyrrolidone coated silver nanoparticles in monovalent and divalent electrolyte solutions. Environmental Science \& Technology, 45(13), 5564-5571.

38. Patterson, K. Y., Pehrsson, P. R.,\& Perry, C. R. (2013). The mineral content of tap water in United States households. Journal of Food Composition and Analysis, 31(1), 46-50.

39. Tuzimski, T.,\& Sherma, J. (2015) High performance liquid chromatography in pesticide residue analysis.1st ed., CRC Press, Boca Raton, FL, USA.

\section{Figures}


a

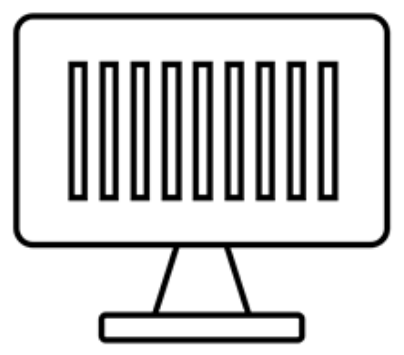

b

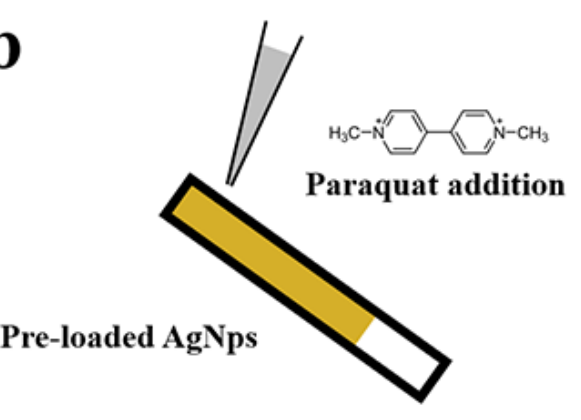

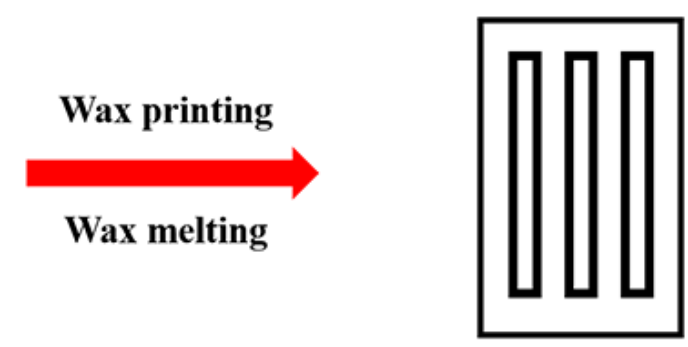

Hydrophillic channel with Hydrophobic border

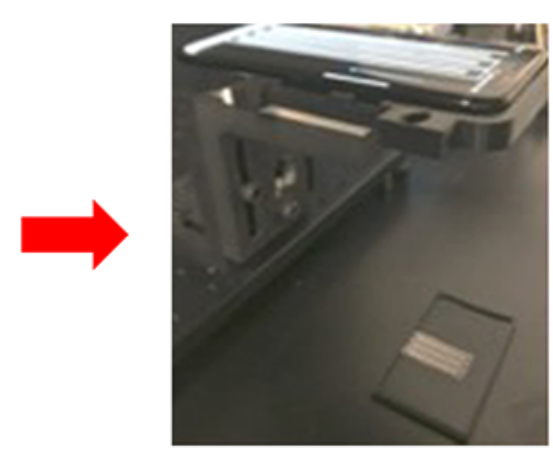

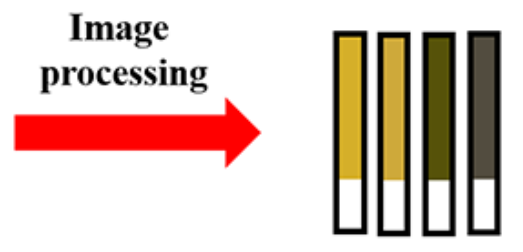

Analysis of colorimetric information (RGB intensity)

Photographic system

Figure 1

Schematic illustrations of (a) $\mu P A D$ fabrication and (b) smartphone-based paraquat detection from AgNP-loaded $\mu$ PAD. 
a
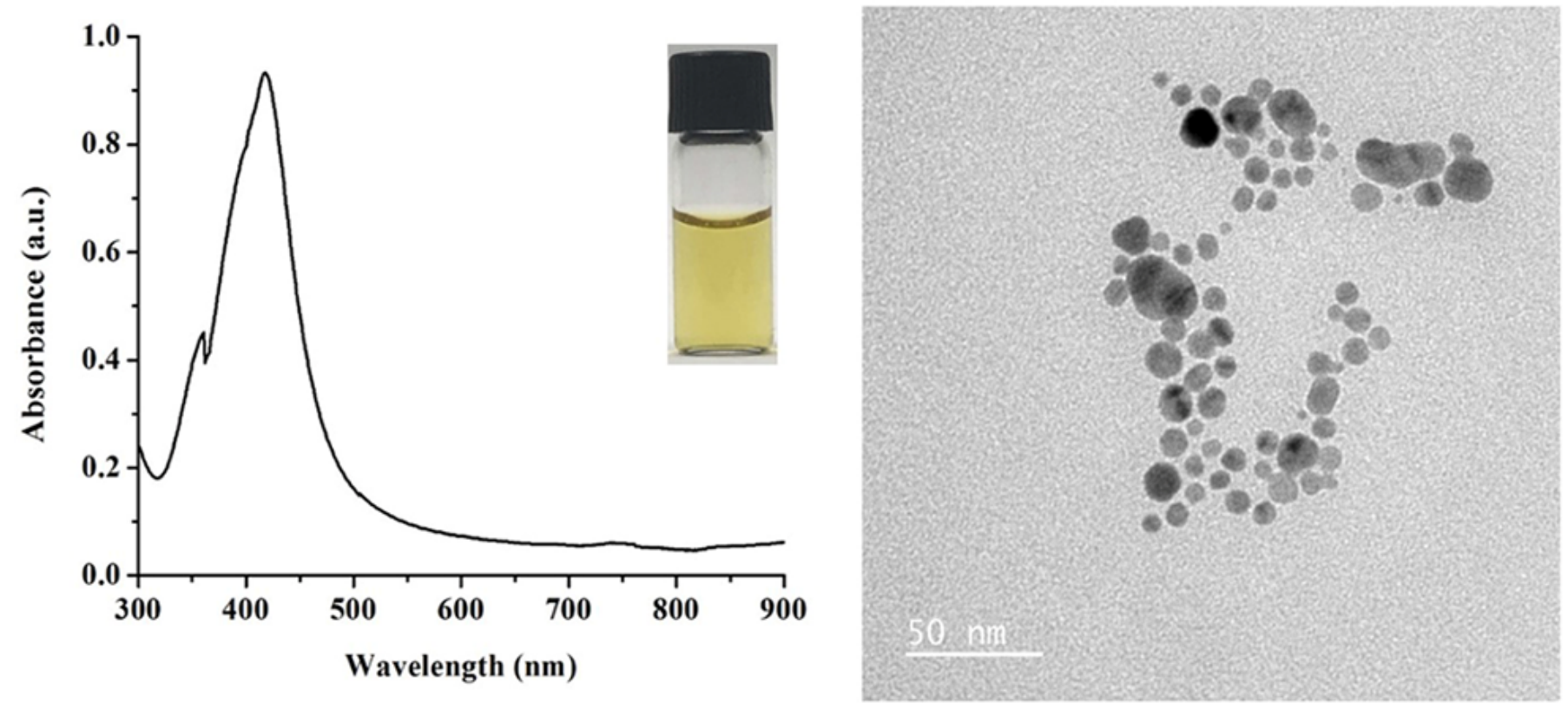

C
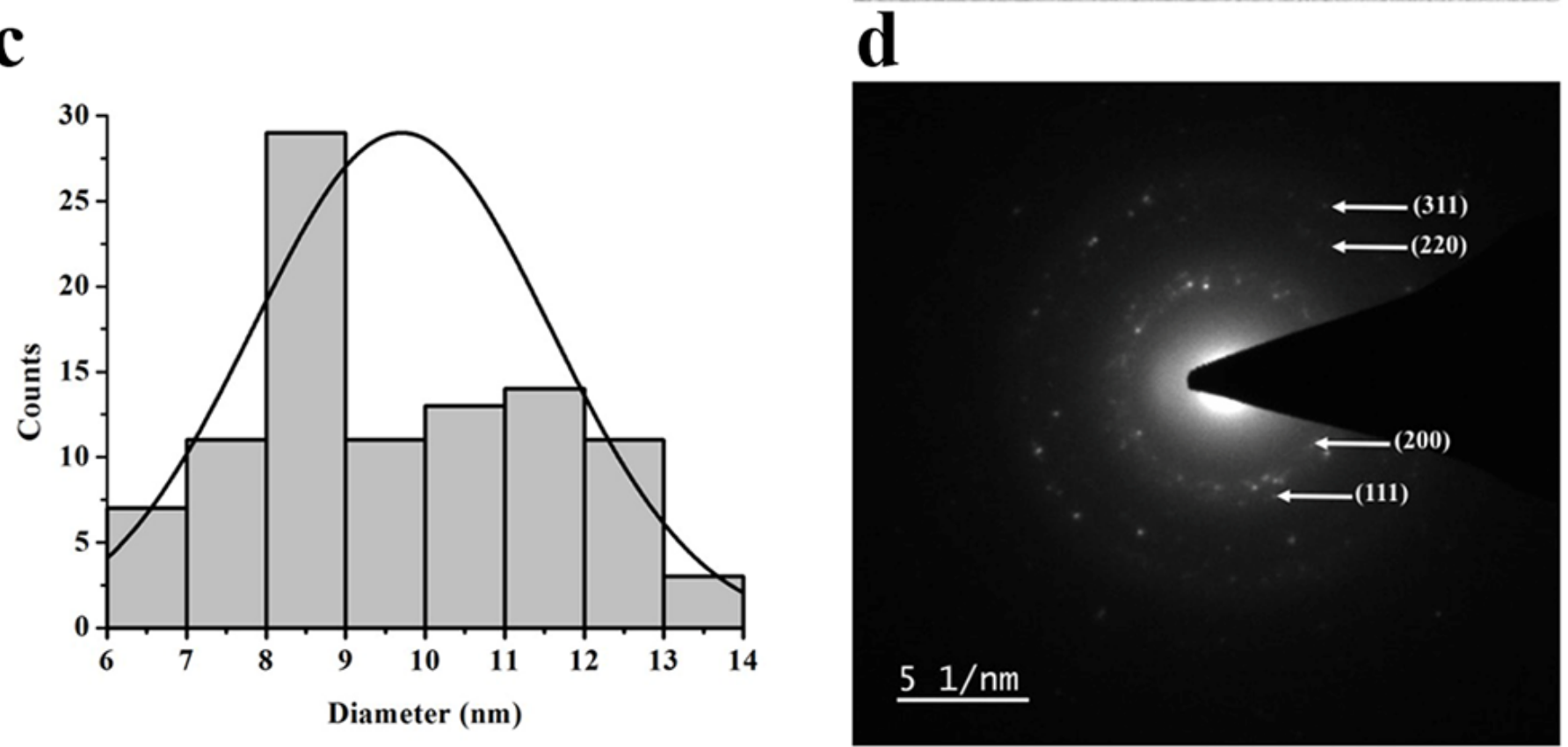

Figure 2

Characterization of the synthesized AgNPs as analyzed by (a) UV-Vis spectrum, (b) TEM image, (c)size distribution from TEM images, and (d) SAED pattern. 


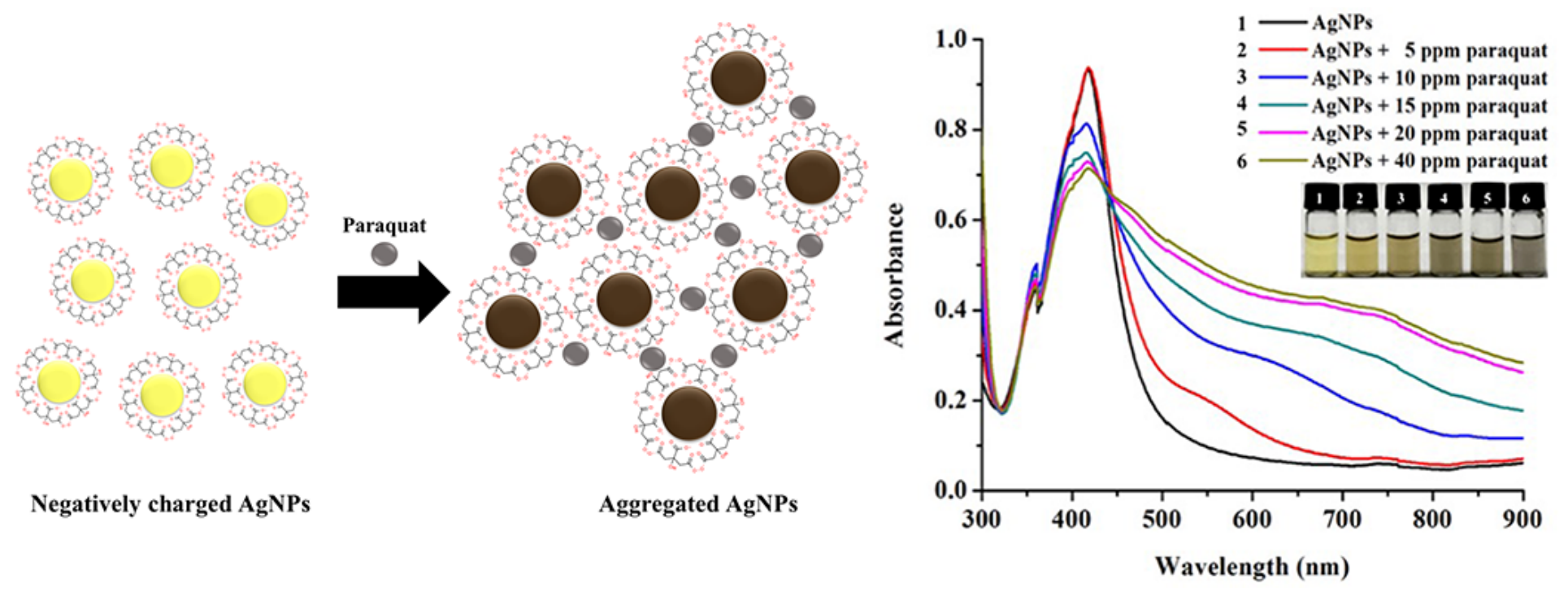

Figure 3

Images of(a) the mechanism of paraquat detection withcitrate-capped AgNPs, and (b) the UV-vis absorbance spectra of AgNPs with varying concentrations of paraquat. 
a

\section{Concentration (ppm)}

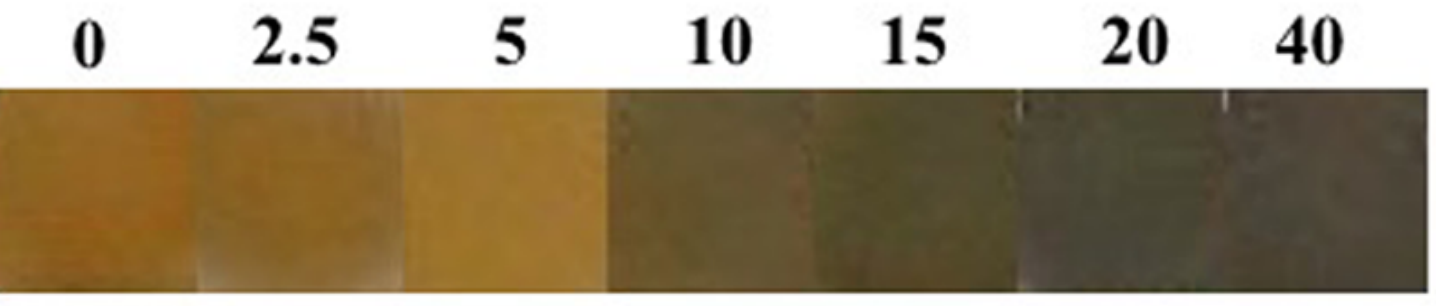

b

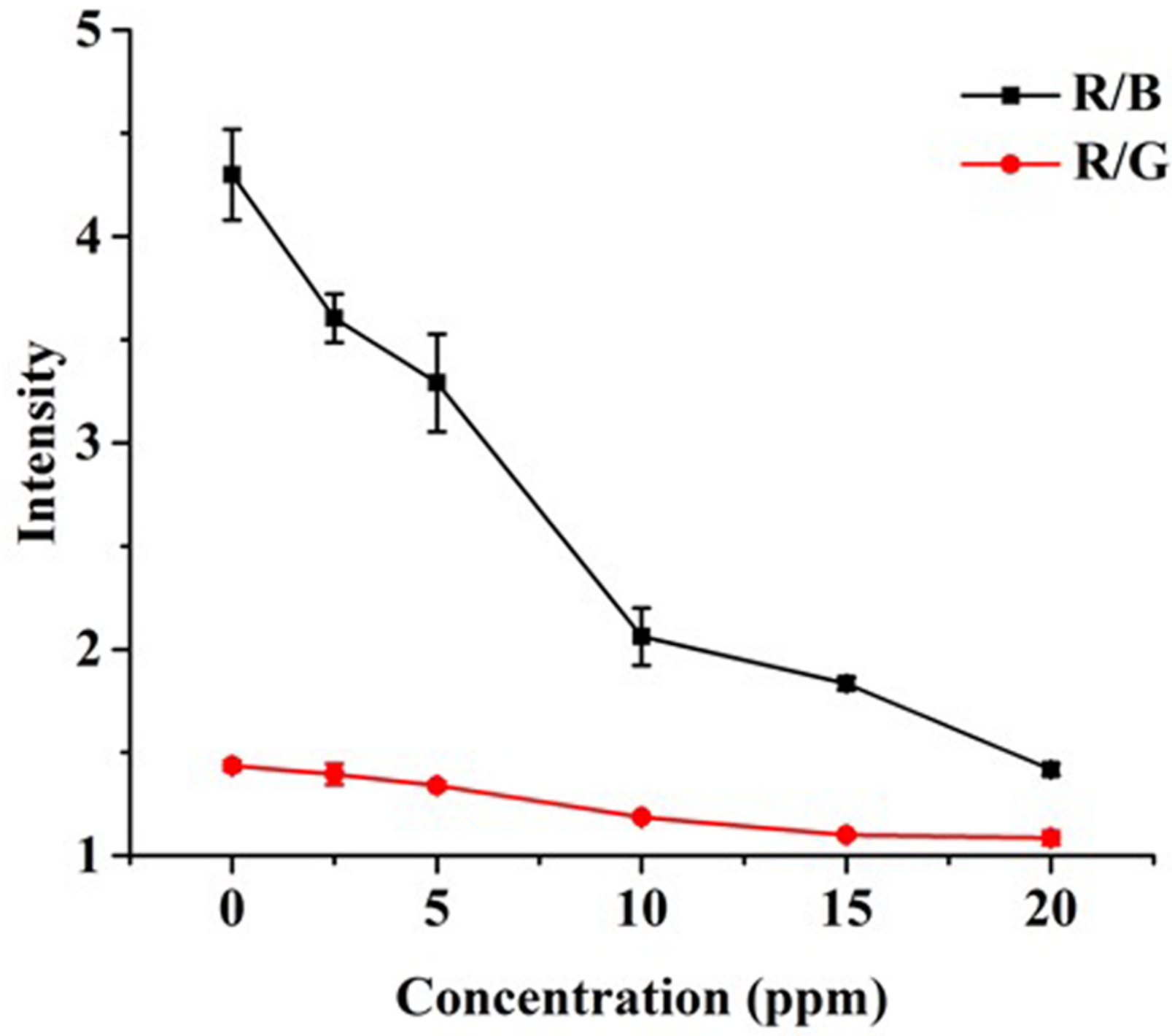

Figure 4

Images of(a) the $\mu$ PAD channels showing different colorations of AgNPs with respect to varying concentrations of paraquat, and (b) the R/B (red to blue) and R/G (red to green) intensities obtained from (a). Averages from three different experiments, each time on a different $\mu$ PAD channel. Error bars represent standard deviations. 


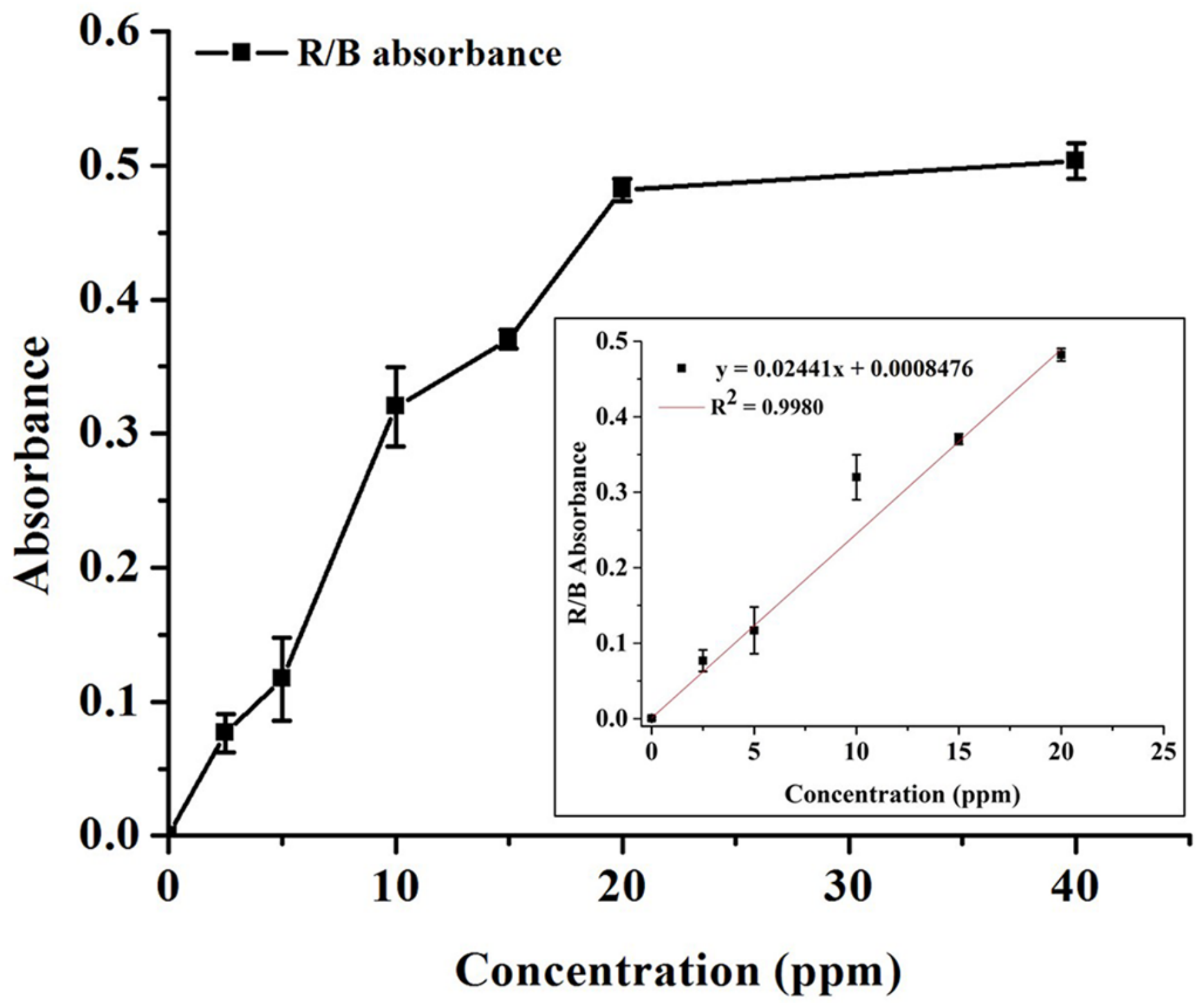

Figure 5

Mean absorbance values obtained from R/B intensities on $\mu \mathrm{PAD}$ are plottedagainst paraquat concentrations to construct a calibration curve. Averages from three different experiments, each time on a different $\mu \mathrm{PAD}$ channel. Error bars represent standard deviations. 


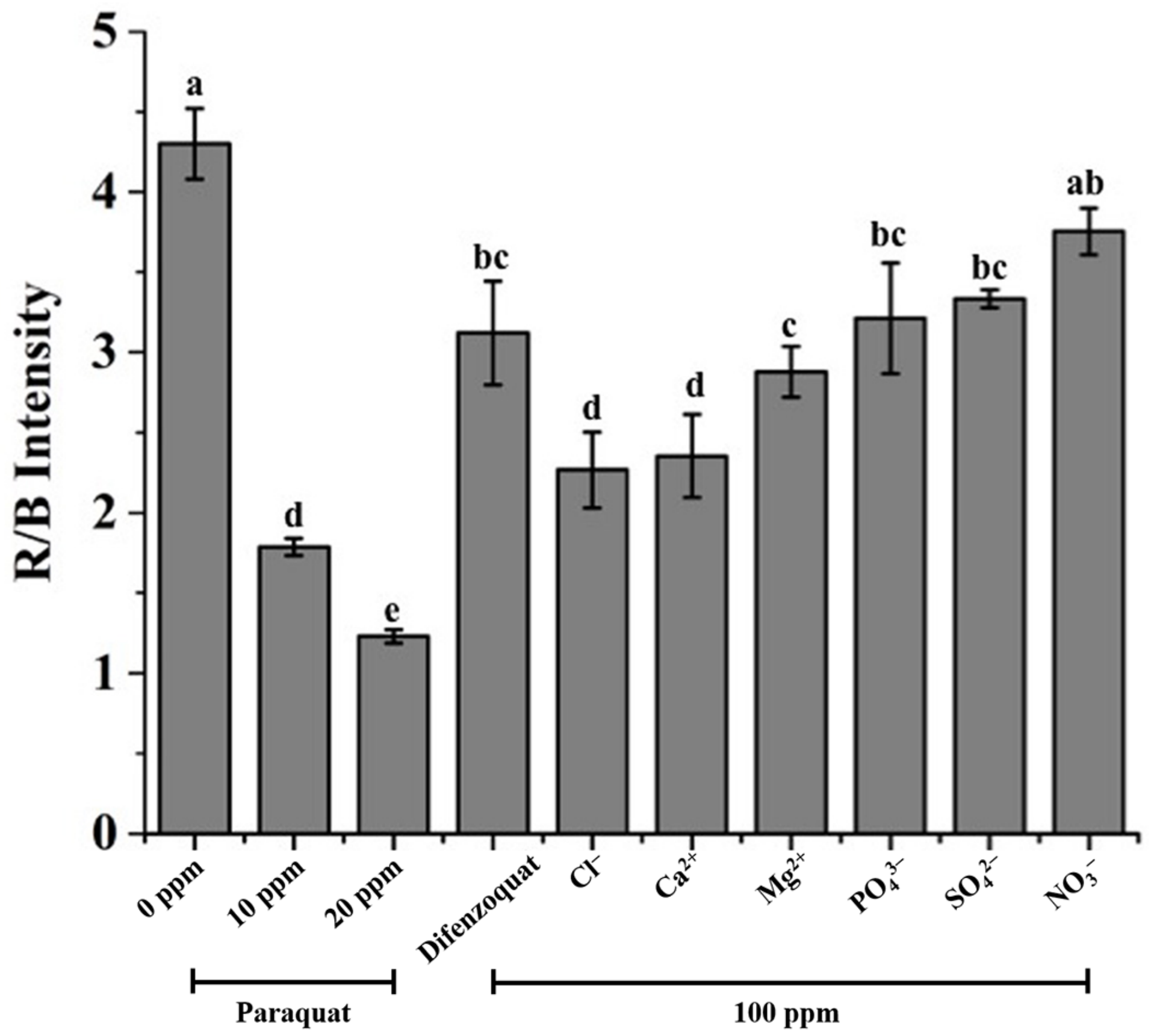

Figure 6

MeanR/B intensities on $\mu$ PAD for DI water (control), paraquat (10 and $20 \mathrm{ppm}$ ), andotherforeign substances $(100 \mathrm{ppm})$. Averages from three different experiments, each time on a different $\mu P A D$ channel. Error bars represent standard deviations. 